\title{
Ovarialkarzinom: Chemotherapie intraperitoneal?
}

\begin{abstract}
Die intraperitoneale Applikation der Chemotherapie bei fortgeschrittenem Ovarialkarzinom wird nicht routinemäßig eingesetzt. Anhand gepoolter Daten der GOG (Gynecologic Oncology Group) wurde der Langzeiterfolg überprüft.
\end{abstract}

_ ür ihre retrospektive Post-hoc-Studie - verwendeten US-amerikanische Ärzte die Ergebnisse der Studien GOG 114 (ursprünglich 589 Patientinnen) und GOG 172 (429 Patientinnen). Bei den Patientinnen mit fortgeschrittenem Ovarialkarzinom im FIGO-Stadium III hatten mögliche Resttumoren nach der Operation höchstens $1 \mathrm{~cm}$ Durchmesser.

In der GOG-114-Studie erhielten sie entweder Paclitaxel intravenös (i.v.) und danach Cisplatin i.v. über insgesamt 6 Zyklen oder Carboplatin i.v. über 2 Zyklen und danach Paclitaxel i.v. an Tag 1 der Behandlung und am 8. Tag Cisplatin intraperitoneal (i.p.) über insgesamt 6 Zyklen. In der Studie GOG 172 wurde den Patientinnen zunächst Paclitaxel i.v. und danach am 2. Tag Cisplatin i.v. verabreicht. Vergleichsgruppe waren Patientinnen, die an Tag 2 Cisplatin i.p. und ebenso i.p. Paclitaxel am 8. Tag über 6 Zyklen bekamen. Das mediane Follow-up lag bei fast 11 Jahren.

Die Auswertung der gepoolten Daten ergab eine mediane Überlebenszeit bei Patientinnen mit i.p.-Applikation von 62 Monaten (95\%-Konfidenzintervall [95\%-KI] 55,5-69,5 Monate). In der Vergleichsgruppe mit i.v.-Verabreichung der Chemotherapie lag sie mit 51 Monaten (95\%-KI 46,0-58,2 Monate) deutlich niedriger (adjustierte Hazard Ratio [HR] 0,77; $95 \%$-KI 0,65-0,90; $\mathrm{p}=0,002)$. Die Wahrscheinlichkeit der Krankheitsprogression wurde im Vergleich zur i.v.Behandlung um etwa $21 \%$ verringert (adjustierte HR 0,79; $95 \%$-KI 0,67-0,92; $\mathrm{p}=0,003)$. Herausgerechnet wurden jeweils demografische und mehrere klini- sche und pathologische Parameter, etwa Alter und Histologie.

Schlechter schnitten in beiden Therapiegruppen u.a. Patientinnen ab, die weniger Chemotherapiezyklen hatten oder bei denen eine größere Tumorrestmenge vorlag. So nahm z. B. das Sterberisiko mit jedem i.p.-Therapiezyklus um $12 \%$ ab (adjustierte HR 0,88; $95 \%$-KI 0,83 und $0,94 ; \mathrm{p}<0,001)$.

Fazit: Der Vorteil einer intraperitonealen Chemotherapieapplikation lässt sich bei Frauen mit fortgeschrittenem Ovarialkarzinom offenbar auch langfristig erkennen. Vor einer routinemäßigen Anwendung außerhalb von Studien sind jedoch noch die Ergebnisse mehrerer derzeit laufender Studien abzuwarten, etwa der Studie GOG 0252 zum Effekt einer zusätzlichen Angiogenesehemmung.

Peter Leiner

Tewari D et al. Long-term survival advantage and prognostic factors associated with intraperitoneal chemotherapy treatment in advanced ovarian cancer: a gynecologic oncology group study. J Clin Oncol. 2015;33(13):1460-6.

\section{Brachytherapie bei inoperablem Stadium-I-Endometriumkarzinom}

\section{Leitlinien zum Vorgehen bei inoperablem Endometriumkarzinom im Stadium I existieren zurzeit nicht. Was nutzt eine Brachytherapie mit oder ohne externer Strahlenthera- pie? Daten einer Registerstudie geben Auskunft.}

\footnotetext{
- twa $10 \%$ der Patientinnen mit einem - Endometriumkarzinom im FIGOStadium I sind bereits nicht mehr operabel. Als Gründe spielen vor allem Übergewicht und damit verbundene Komorbiditäten wie z. B. Diabetes mellitus eine Rolle. Für diese Patientinnen existiert bisher noch kein Standardvorgehen. Kliniker berücksichtigen in den Therapieplänen häufiger eine Brachytherapie, wenngleich deren Erfolg bisher aufgrund nur kleinerer Studien nicht gut abgesichert ist. In einer populationsbasierten Datenbankanalyse sollte nun der Nutzen einer Brachytherapie in größerem Stil
}

überprüft werden. US-amerikanische Onkologen werteten dazu retrospektiv die Daten von 18 Registern aus, die im Rahmen des Programms „Surveillance, Epidemiology, and End Results“ (SEER) des National Cancer Institute von 1998 bis Ende 2011 angefallen waren.

Für die Auswertung zur Verfügung standen die Angaben zu insgesamt 460 Frauen mit einem Endometriumkarzinom im Stadium I, die nicht operiert wurden und eine Strahlentherapie erhielten - entweder nur eine externe Radiotherapie ( $n=260)$ oder eine Brachytherapie mit oder ohne externer Strahlentherapie $(n=200)$.

Als einzigen Faktor, der signifikant mit dem Einsatz einer Brachytherapie verbunden war, identifizierten die Statistiker ein jüngeres Patientenalter (median 72 vs. 76 Jahre, $p=0,001$ ). Bei den brachytherapierten Frauen ergaben sich signifikant höhere Raten für das 3-Jahres-Gesamtüberleben (60 vs. $47 \%$; $\mathrm{p}<0,001)$ sowie das krankheitsspezifische Überleben ( 82 vs. $74 \% ; \mathrm{p}=0,032$ ). Diese Überlegenheit der Brachytherapie ggf. in Kombination mit externer Strahlentherapie im Vergleich zu einer alleinigen externen Bestrahlung zeigte sich auch in multivariaten sowie alters- und Grading-abgeglichenen Analysen.

Fazit: Patientinnen mit einem inoperablen Endometriumkarzinom im Stadium I profitieren von einer Brachytherapie und eventuell einer zusätzlichen externen Strahlentherapie unabhängig vom Alter und Tumorgrad. Sowohl das krankheitsfreie als auch das Gesamtüberleben sind im Vergleich zu lediglich extern bestrahlten Frauen deutlich besser.

Barbara Kreutzkamp

Acharya S et al. Brachytherapy is associated with improved survival in inoperable stage I endometrial adenocarcinoma: A population-based analysis. Int J Radiat Oncol Biol Phys. 2015 June 15. [Epub ahead of print]. 